\title{
Assessment of patient pain at colonoscopy: are nurses better than endoscopists?
}

\author{
S Ramakrishnan MB MRCP JY Yiannakou MB MRCP W R Ellis DM MRCP I M Bain MB FRCS
}

J R Soc Med 2004;97:432-433

\section{SUMMARY}

The pain that patients recollect having experienced at colonoscopy is likely to influence uptake of the procedure. We used visual analogue scales to assess recollected pain shortly before discharge, and compared these scores with assessments by the endoscopist and the attending nurse.

Data were complete for 426 procedures (90\%). The mean perceived pain score for patients was 3.2 , for endoscopists 2.8 and for nurses 3.1. On multivariate analysis, the endoscopists' assessments of pain had little predictive value over and above those of nurses, whereas nurses' assessments remained significant when adjusted for endoscopists' assessments.

Nurses were more accurate than endoscopists in gauging the pain of colonoscopy. This may be because endoscopists are focused on the video monitor while nurses are focused on the patient. More active use of nurses' assessments might help keep pain to a minimum.

\section{INTRODUCTION}

Most patients expect a colonoscopy to be painful, and this reputation adversely affects uptake. Moreover, the patient who remembers a painful colonoscopy may decline to have a repeat procedure. This matter will become even more relevant in the UK if a national screening programme for colon cancer is instituted. Previous studies have shown disparities between patients' reported pain at colonoscopy and the pain that medical staff perceived them to have experienced. ${ }^{1-5}$ However, the numbers were small and the investigations were subsidiary to the main purpose of the studies. We have looked prospectively at the performance of endoscopists and nurses in assessing pain during colonoscopy.

\section{METHOD}

Data were collected prospectively for twelve months during which 474 patients agreed to undergo outpatient colonoscopy in our district general hospital. All those who could complete a simple questionnaire were included. At the start of the procedure patients received intravenous midazolam and Buscopan (hyoscine butylbromide), and pethidine was used as an analgesic at the discretion of the endoscopist.

Pain scores were recorded on a visual analogue scale of $0-10$ on which 0 represented no pain and 10 the worst

Department of Gastroenterology, University Hospital of North Durham, Durham DH1 5TW, UK

Correspondence to: Dr S Ramakrishnan

E-mail: subramaniamram@yahoo.co.uk imaginable pain. Just before discharge from the endoscopy unit patients recorded the pain they had experienced during the procedure. Pain scores as assessed by nurses and endoscopists were recorded soon after completion of the procedure. All were blind to the others' scoring.

\section{Box 1 Demographic data and clinical details}

\begin{tabular}{lc|} 
Variable & \\
Age range (years) & $17-88$ \\
Mean age (years) & 59 \\
Completion rate (\%) & 89 \\
Indication for colonoscopy & \\
Diarrhoea/altered bowel habit (\%) & 26 \\
Abdominal pain (\%) & 2 \\
Bleeding (\%) & 10 \\
Cancer/polyp follow-up (\%) & 24 \\
Iron deficiency anaemia (\%) & 16 \\
IBD-related (\%) & 13 \\
Other (\%) & 9 \\
Findings & \\
Normal (\%) & 45 \\
Polyp (\%) & 19 \\
Colitis (\%) & 11 \\
Cancer (\%) & 4 \\
Angiodysplasia (\%) & 2 \\
Diverticula (\%) & 8 \\
Other (\%) & 10 \\
\hline
\end{tabular}

$\mathrm{IBD}=$ inflammatory bowel disease 


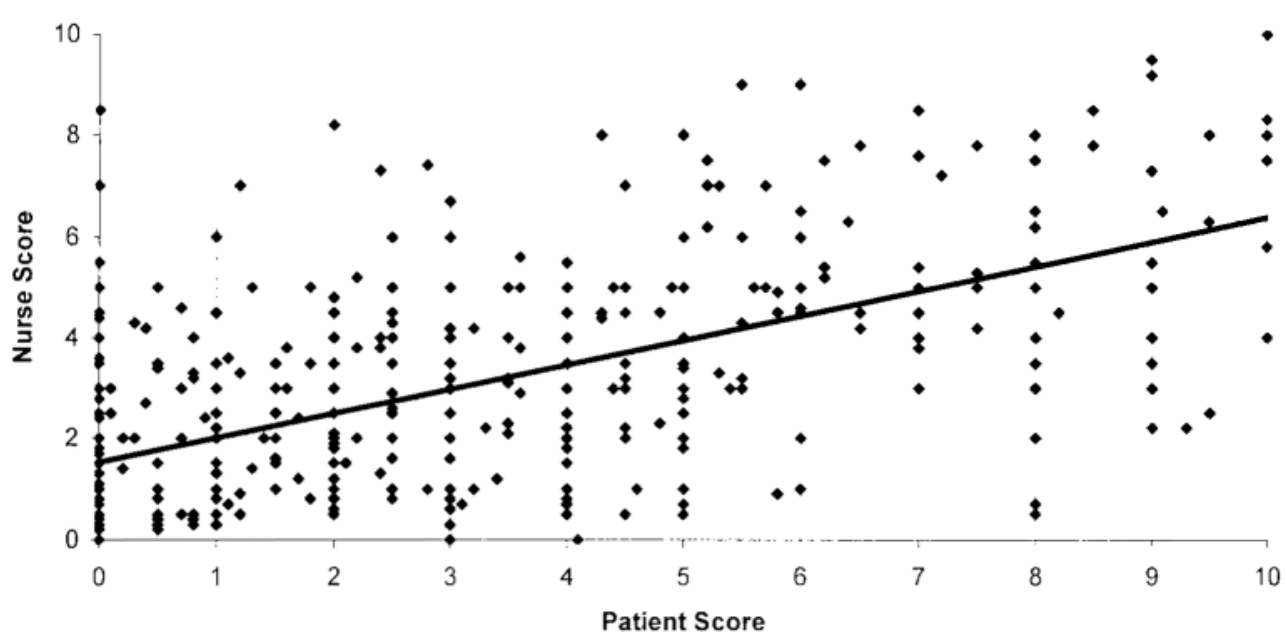

Figure 1 Correlation of pain score: nurse with patient

Pain scores were expressed as mean and standard error (SE). Complete data were assessed by multivariate linear regression analysis.

\section{RESULTS}

Data were complete for 426 (90\%) of the procedures: Box 1 summarizes the indications and results. Patients' mean perceived pain score was 3.2 (SE 0.13). The pain score assessed by the endoscopist was $2.8(0.1)$ and that assessed by the nurse was $3.1(0.1)$. Comparison of pain scores of endoscopists and nurses with those of patients gave correlation coefficients of 0.42 and 0.59 , respectively. Figure 1 indicates that, even for nurses, there was much scatter. On multivariate analysis, endoscopists' assessments were no longer significant when adjusted for nurses' assessments $(P=0.39)$ whereas nurses' assessments were significant when adjusted for endoscopists' assessments $(P<0.01)$.

\section{DISCUSSION}

The main point of interest from this study derives from the multivariate analysis, indicating that endoscopists could reach a more accurate assessment of the patient's pain by consulting the nurse, whereas the reverse was not true. We must, however, acknowledge a weakness of research of this kind - that the effect of sedation and analgesia could have influenced not only the pain assessments by the endoscopists and nurses but also the recollection of perceived pain by the patients. In the case of the health workers, the assessments do represent a collective judgment on the pain experienced by the patient throughout the procedure irrespective of the doses of sedation and analgesia used.

Why should nurses outperform endoscopists in this respect? We suspect it is because endoscopists are procedure-focused, aiming to complete the procedure successfully in a high proportion of patients. Nurses on the other hand are mainly patient focused. We suggest, therefore, that nurses' assessments should be actively used during colonoscopy, to achieve the best possible compromise between success rate and patient discomfort.

\section{REFERENCES}

1 Hull T, Church JM. Colonoscopy - how difficult, how painful? Surg Endosc 1994;8:784-7

2 Nivatvongs S. How painful is colonoscopy? Gastrointest Endosc 1991;27:127

3 Ristikarante M, Hartikainen J, Heikkinen M, Janatuinen E, Julkunen R. The effects of gender and age on colonoscopic examination. $J$ Clin Gastroenterol 2001;32:69-75

4 Kim LS, Koch J, Yee J, Halvorsen R, Cello JP, Rockey DC. Comparison of patients' experiences during imaging tests of the colon. Gastrointest Endosc 2001;54:67-74

5 Redelmeier DA, Kahneman D. Patients' memories of painful medical treatments: real time and retrospective evaluations of two minimally invasive procedures. Pain 1996;66:3-8 